\title{
THE
}

2010

\section{Nuchal Cord Management and Nurse-Midwifery Practice}

Judith S. Mercer

University of Rhode Island, jsmercer@uri.edu

Rebecca L. Skovgaard

Joann Peareara-Eaves

Tracey A. Bowman

Follow this and additional works at: https://digitalcommons.uri.edu/nursing_facpubs

Terms of Use

All rights reserved under copyright.

\section{Citation/Publisher Attribution}

Mercer, J. S., Skovgaard, R. L., Peareara-Eaves, J., \& Bowman, T. A. (2010). Nuchal Cord Management and Nurse-Midwifery Practice. Journal of Midwifery \& Women's Health, 50(5), 373-379. https://doi.org/ 10.1016/j.jmwh.2005.04.023

Available at: https://doi.org/10.1016/j.jmwh.2005.04.023

This Article is brought to you for free and open access by the College of Nursing at DigitalCommons@URI. It has been accepted for inclusion in College of Nursing Faculty Publications by an authorized administrator of DigitalCommons@URI. For more information, please contact digitalcommons-group@uri.edu. 


\title{
Nuchal Cord Management and Nurse-Midwifery Practice
}

\author{
Judith S. Mercer, CNM, DNSc, Rebecca L. Skovgaard, CNM, MS, \\ Joann Peareara-Eaves, CNM, MS, and Tracey A. Bowman, CNM, MS
}

\begin{abstract}
Nuchal cord, or cord around the neck of an infant at birth, is a common finding that has implications for labor, management at birth, and subsequent neonatal status. A nuchal cord occurs in $20 \%$ to $30 \%$ of births. All obstetric providers need to learn management techniques to handle the birth of an infant with a nuchal cord. Management of a nuchal cord can vary from clamping the cord immediately after the birth of the head and before the shoulders to not clamping at all, depending on the provider's learned practices. Evidence for specific management techniques is lacking. Cutting the umbilical cord before birth is an intervention that has been associated with hypovolemia, anemia, shock, hypoxic-ischemic encephalopathy, and cerebral palsy. This article proposes use of the somersault maneuver followed by delayed cord clamping for management of nuchal cord at birth and presents a new rationale based on the available current evidence. J Midwifery Womens Health 2005;50:373-379 (C) 2005 by the American College of Nurse-Midwives.
\end{abstract}

keywords: nuchal cord, somersault maneuver, umbilical cord clamping, resuscitation

\section{INTRODUCTION}

\section{“...the practice of severing the umbilical cord prior to the delivery of the body of the fetus, proposed in some textbooks as routine procedure, is a dangerous technique, which should be avoided."}

Umbilical cord around the neck of an infant, or nuchal cord, may affect the infant's status during labor, at birth, and after birth. Nuchal cords occur in $20 \%$ to $30 \%$ of births, ${ }^{2-4}$ requiring that all providers learn management techniques to handle the birth of an infant with a nuchal cord. However, evidence is lacking to support a provider's choice in management techniques.

Management of a nuchal cord varies. Some providers clamp the cord immediately after the birth of the head and before the shoulders, whereas others do not clamp at all. Standard medical management recommends one attempt to slip the cord over the infant's head just before delivery of the body or, should the cord be too tight, to clamp and cut prior to delivery of the shoulders. ${ }^{5}$

Although most infants appear to tolerate this process with minimal distress, there is evidence that cutting the cord before delivery of the shoulders can result in neonatal morbidity and even mortality. ${ }^{1}$ A review of the literature on nuchal cord reveals that it is associated with increased risk for hypovolemia, ${ }^{6}$ anemia, ${ }^{7}$ cerebral palsy, ${ }^{8,9}$ and even death. ${ }^{2}$ Iffy proposes that it is the cutting of the cord before birth of the shoulders that leads to hypoxic-ischemic encephalopathy and recommends the use of the somersault maneuver, ${ }^{10,11}$ which keeps the cord intact during this critical time. ${ }^{1}$ This simple procedure involves somersaulting the infant's head toward the mother's thigh as the infant emerges, immediately unwinding the cord, and allowing the infant to reperfuse.

Address correspondence to Judith S. Mercer, CNM, DNSc, FACNM, NurseMidwifery Program, University of Rhode Island College of Nursing, 2 Heathman Road, Kingston, RI 02881-2021. E-mail: jmercer@uri.edu
The purpose of this article is to encourage the use of somersault maneuver for management of nuchal cord at birth and offer a sound rationale for its use based on the available current evidence. The anatomy and physiology related to the nuchal cord are reviewed, followed by neonatal transitional physiology and the literature on nuchal cord and immediate cord clamping. A discussion of management options offers the somersault maneuver as the preferred practice in the presence of a nuchal cord to prevent disruption of the physiologic neonatal transition and avoid neonatal morbidity and mortality. ${ }^{10}$

\section{NUCHAL CORD PHYSIOLOGY}

Interventions during birth should be based first on thorough understanding of relevant concepts of physiology to build one's rationale for practice. In nuchal cord management, the relevant physiologic mechanisms include properties related to nuchal cord, physiology of the fetal-placental circulation, and neonatal transitional physiology.

\section{Incidence and Etiology of Nuchal Cords}

Nuchal cord occurs in approximately $25 \%$ of births. ${ }^{3}$ Recent prenatal ultrasonographic evaluation reveals nuchal cords are dynamic in nature, forming and resolving over the course of a pregnancy. ${ }^{12}$ Even at 10 to 14 weeks' gestation, $8 \%$ of fetuses may have nuchal cords. ${ }^{13}$ The occurrence of nuchal cord increases with gestational age; at 42 weeks, $29 \%$ of births have nuchal cord present. ${ }^{2}$

The mean length of the umbilical cord is approximately $60 \mathrm{~cm}$ (range 50 to $70 \mathrm{~cm}$ ). ${ }^{2}$ Nuchal cords occur more frequently among fetuses with longer cords. In utero, the umbilical cord appears to lengthen in response to tensile forces caused by fetal movement. ${ }^{12}$ Maximal cord length appears to be achieved around 30 weeks' gestation, with little growth in length beyond that time. ${ }^{12}$ Fetuses in vertex presentations have been found to have longer cords than those in breech presentations. 


\section{Structure of the Umbilical Cord}

The umbilical cord usually consists of two arteries and one vein, surrounded by Wharton's jelly, and enclosed in a thin layer of amnion. An extensive network of collagen fibrils within Wharton's jelly forms a "soft skeleton" surrounding the umbilical vessels and provides some protection and support. ${ }^{14}$ However, it is the high venous and arterial pressures in the blood flow that keeps the umbilical cord distended and prevents interruption of blood flow even during compression or torsion. ${ }^{15}$ The collapsed vessels typically seen on inspection after ligation at birth are misleading. The vessels with intact fetoplacental circulation are distended and take up the greatest portion of the cross-sectional area of the cord. ${ }^{15}$ The arteries are narrower in diameter than the vein, having both more muscular and thicker walls. They maintain a pulsatile flow of blood at systolic and diastolic pressures of about 60 and $30 \mathrm{~mm} \mathrm{Hg}$, respectively. Blood flow through the relatively larger, thin-walled umbilical vein is much less pulsatile, at a pressure of about 20 to $25 \mathrm{~mm} \mathrm{Hg}$.

\section{Effects of Pressure}

In the fairly closed system of the intrauterine environment, pressure is more or less evenly distributed, and the freefloating cord is not greatly affected by the increase in pressure brought about by contractions. However, segments of cord encircling the fetal neck or other body part cannot float freely and may be compressed by the substantial pressure from the contracting uterine musculature. The vein is easier to occlude than the arteries, and compression commonly results in variable decelerations of the fetal heart rate that, when severe, may affect fetal tolerance of labor. ${ }^{16}$

When the umbilical vein is regularly occluded, there is a net transfer of blood from the fetus to the placenta. As pressure on the cord and compression increases, the muscular-walled, high-pressure arteries may continue to move blood from the fetus to the placenta, whereas return flow to the fetus in the thin-walled vein is impeded. The loss of fetal blood volume may be particularly severe when the recovery time between contractions is short. Neonatal status may be compromised by the uncorrected physiologic effects of hypoxia as well as reduced blood volume.

Judith S. Mercer, CNM, DNSc, FACNM, is a faculty member of the University of Rhode Island College and Principal Investigator for a randomized controlled trial on delayed cord clamping in preterm infants funded by National Institutes of Health.

Rebecca Skovgaard, CNM, MSN, is an assistant professor of clinical obstetrics and gynecology, University of Rochester School of Medicine and Dentistry.

Joann Peareara-Eaves, CNM, MSN, is a 2004 graduate from the University of Rhode Island.

Tracey A. Bowman, CNM, MSN, is an expert in the use of water birth; in her own practice, she provides gynecologic care and a homebirth service.

\section{Fetal Heart Rate Changes With Nuchal Cord}

The fetal heart rate variable decelerations commonly attributed to cord compression can occur as a result of umbilical vessel occlusion. According to Weiss et al., ${ }^{17}$ Doppler studies have demonstrated that, for many variable decelerations, a sharp decrease in umbilical vessel perfusion precedes the drop in the fetal heart rate by a few seconds. The interruption of umbilical perfusion stimulates discharge of the parasympathetic nervous system via the vagus nerve and results in fetal bradycardia.

\section{Other Important Factors}

Factors, such as amniotic fluid volume, the number of encirclements, presence of knots, and tightness of the cord may influence the impact of nuchal cords on fetuses and newborns. Strong et al. ${ }^{18}$ found that oligohydramnios worsened the effect of nuchal cords on fetal status during labor. The same appears to be true of multiple nuchal cord encirclements. ${ }^{4}$

\section{FETAL TO NEONATAL TRANSITION}

To protect the physiologic processes at birth, one must understand the role that blood volume plays in the success of the fetus-to-neonate transition. The following model explains how an adequate blood volume promotes a normal physiologic neonatal transition and helps to protect the infant from harm.

\section{Blood Volume Model of Neonatal Transition}

A shift is needed in thinking about the fetus-to-neonate transition, from the current focus on immediate respiration only to the role that blood volume plays in a successful transition. ${ }^{19}$ The blood volume model states that the limiting factor at birth may be the availability of adequate red blood cells and volume for tissue oxygen delivery. ${ }^{19}$

During fetal life, only $8 \%$ to $12 \%$ of the fetal cardiac output goes to the fetal lungs, whereas $45 \%$ to $50 \%$ circulates through the placenta. ${ }^{20}$ The alveoli during fetal life are filled with lung fluid. Immediately after birth, the lung must structurally change from a fluid-filled organ to one filled with air. Functionally, it must change from an organ of fluid production to an organ of gas exchange. Immediately after birth, $50 \%$ of the neonate's cardiac output must flow to and through the lungs to effect appropriate gas exchange. A volume of blood (at least 40 $\mathrm{mL}$ ) must be available to expand the pulmonary capillary bed. ${ }^{21}$ However, the volume of blood available to the baby for this purpose is limited to the amount that was in the neonate at the time the cord was clamped. If the blood volume needed to expand the lung does not come from the placenta through an intact umbilical cord, it must be forfeited from other organ systems and the general circulation in the neonate's body. ${ }^{22}$ 


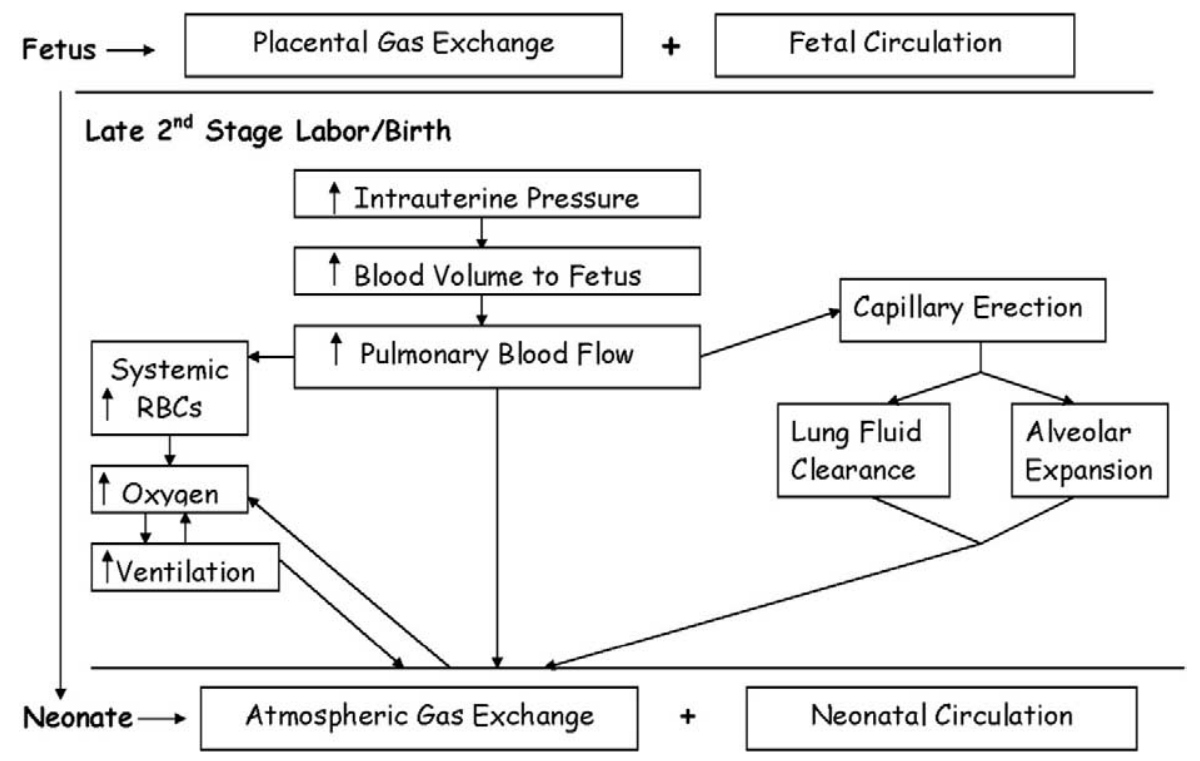

Figure 1. The blood volume model of neonatal transition.

A full-term fetus has approximately 110 to $115 \mathrm{~mL} / \mathrm{kg}$ of blood that is distributed in the fetal and placental compartments. Prior to birth, about two-thirds volume perfuses the fetal body, whereas one third flows through the placenta. A $3000 \mathrm{~g}$ fetus will have approximately 330 to $345 \mathrm{~mL}$ of blood in the fetal-placental circulation. The average blood volume of infants after immediate cord clamping is 70 $\mathrm{mL} / \mathrm{kg}$ versus $90 \mathrm{~mL} / \mathrm{kg}$ in infants after delayed cord clamping. For the $3000 \mathrm{~g}$ infant, this difference is $210 \mathrm{~mL}$ versus $270 \mathrm{~mL}$ for the circulating blood volume. ${ }^{23}$ Delayed cord clamping will provide an extra $60 \mathrm{~mL}$ of blood to the infant for circulatory adjustments. This $60 \mathrm{~mL}$ of blood is needed for lung volume expansion as the neonatal cardiac output to the lung increases from $40 \%$ to $50 \%$ of the cardiac output, in contrast to the fetal flow of $8 \%$ to $12 \% .^{20}$

The millions of capillaries covering the alveoli are actually cemented to the alveoli by an intracellular matrix. ${ }^{24}$ At birth, these capillaries fill with blood for the first time, causing the capillary plexuses to expand with blood and become erect. This process of capillary "erection" opens the alveoli and provides the "scaffolding" structure to keep them open. ${ }^{25}$ Air pressure does not keep lungs open, because lungs have only atmospheric pressure. It is the hydrostatic exoskeleton generated by the capillary network that maintains alveolar expansion and prevents the alveoli from closing or collapsing on expiration. Surfactant helps keep the alveoli open due to reduction in the surface tension, but surfactant does not directly support the alveolar structure. Adequate blood flow to the lung clears the lung fluid during the initial breaths because higher colloidal osmotic pressure of the blood in the capillaries draws the fluid from the alveoli. As respiration continues, a higher level of systemic oxygen stimulates the respiratory centers of the brain and causes the oxygen-sensitive umbilical arteries and ductus arteriosus to close. ${ }^{26}$ Normal neonatal respiration and circulation is then established (Figure 1).

Stembera and colleagues documented that blood flow in the umbilical vein continues at an average of $75 \mathrm{~mL} / \mathrm{min}$ for an average of 100 to 120 seconds after birth. ${ }^{27}$ Anatomic sections of the cord after birth show the arteries are open when the cord is clamped immediately and constricted when clamping is delayed for a few minutes. This postnatal placental circulation continues to support the infant during the transition to breathing. Even in compromised infants, Stembera found a flow of about $50 \mathrm{~mL} / \mathrm{kg}$ per minute. ${ }^{28}$

Symptomatic polycythemia and hyperbilirubinemia are the two concerns most commonly associated with delayed cord clamping. The idea that delayed cord clamping causes either problem is based on two reports from a study from the 1960s, which did not randomly allocate subjects and has not been replicated. ${ }^{29,30}$ Systematic reviews of the literature reveal that neither risk has been validated in randomized controlled trials of delayed cord clamping involving preterm or term infants. ${ }^{31-33}$ Further discussion can be found in a comprehensive review of the literature on cord clamping. ${ }^{31}$

\section{LITERATURE REVIEW ON EFFECTS OF NUCHAL CORD}

Immediate cord clamping after birth has been shown to result in a $25 \%$ to $40 \%$ reduction in blood volume at birth. ${ }^{23}$ Clamping the cord before birth removes the infant from the placental life support system before the infant is born and increases blood volume loss. The basic cause of hypoxia is interruption of the normal blood flow between the fetus and 
the placenta. The practice of clamping the cord before birth places the infant at high risk of hypoxia, hypovolemia, and related problems. If there is a delay in delivery of the shoulders, this practice can lead to morbidity and mortality. It also necessitates an abrupt, rather than gradual, adaptation to extrauterine breathing. ${ }^{19,34}$

Potential adverse effects from premature ligation of nuchal cords and the resulting blood loss include hypovolemia, hypotension and shock, ${ }^{6,35}$ anemia, ${ }^{7}$ and cerebral palsy. ${ }^{1,8,9}$ An infant who presents with a nuchal cord may already be compromised because of compression of the umbilical cord during contractions, which prevents normal blood flow and correction of acid-base imbalance. If the cord is not cut before or immediately after birth, the infant may be able to equalize these imbalances after birth when the nuchal cord is reduced.

\section{Hypotension and Hypovolemic Shock}

In 1973, Cashore and Usher reported that tight nuchal cords at birth resulted in neonatal hypovolemia in a case series of 11 infants. ${ }^{6}$ Infants requiring cord ligation prior to delivery of the shoulders had a $20 \%$ reduction of red blood cell volume after birth. The diminished blood flow to the fetus accounted for a decrease in body iron content, resulting in anemic, pale, and hypotensive infants after birth. ${ }^{6}$

In a case report, Vanhaesebrouck described two term infants who suffered from acute hypovolemic shock resulting from a tight nuchal cord, despite unremarkable pregnancies and labors. ${ }^{35}$ Early clamping and cutting of the cords was deemed necessary for delivery because the nuchal cords were too tight to slip over the infants' heads. These infants exhibited pallor, irregular respirations, low Apgar scores, gasping, tachycardia, weak peripheral pulses, hypotension, and acidemia. Resuscitation efforts included intubation and ventilation as well as blood transfusions to restore blood volume. The authors suggested "fetoplacental hemorrhage" due to tight nuchal cord was the cause of the infants' condition.

\section{Anemia}

An observational study by Shepherd examined tight or loose nuchal cord as a potential cause of neonatal anemia in 437 newborns consecutively admitted to the nursery. ${ }^{7}$ Anemia was defined as any venous hemoglobin level less than $13.2 \mathrm{~g} / \mathrm{dL}$ or hematocrit of less than $39.2 \%$. She found that $16 \%$ of 57 neonates with a nuchal cord were anemic within the first 24 hours after birth. Three infants in the nuchal cord group developed hypotension requiring blood transfusions, but no anemia was found in the group without a nuchal cord.

\section{Nuchal Cord and Shoulder Dystocia}

Shoulder dystocia occurs in $1.7 \%$ of births and is most often an unanticipated event. A few case reports suggest that clamping and cutting the nuchal cord before delivery of the infant's shoulders can influence outcomes after shoulder dystocia. Iffy described several cases of cerebral palsy after nuchal cords were cut, and subsequent shoulder dystocia delayed birth by as little as 3 minutes. $^{8}$ All of the fetuses were considered healthy prior to the onset of labor. The infants were all born with low Apgar scores and developed signs of hypoxic-ischemic encephalopathy. The authors highly advise avoiding nuchal cord ligation prior to full delivery whenever possible. Iffy remarked that, in Europe, it is routine to deliver the infant's head and then wait for the next contraction to effect restitution and deliver the shoulders. ${ }^{1}$ The diagnosis of shoulder dystocia is not made until after this second contraction. This is in contrast to the practice in the United States of attempting delivery of the shoulders immediately after delivery of the head.

\section{Reduction in Birth Weight}

Three large studies document reduction in weight of 60 to $80 \mathrm{~g}$ in infants after immediate or prebirth cord clamping, due to either a nuchal cord or immediate clamping during active management of third stage. Records of 10,509 Canadian births were examined and revealed that $25 \%$ (2699) of infants born with a nuchal cord had mean weights about $60 \mathrm{~g}$ less than infants without nuchal cords $(\mathrm{n}=8710$ or $74.3 \%)^{3}{ }^{3}$ Typical practice in these settings when a nuchal cord was noted was to cut and clamp the cord before the body of the baby is delivered. This reduction in birth weight is similar to that found in the Hinchingbrooke and Bristol Active Management of Third Stage Trials. ${ }^{36,37}$ Both of these randomized controlled trials studied the incidence of maternal and fetal morbidity after routine active management of the third stage of labor versus expectant or physiologic management. Their definition of active management of third stage included immediate cord clamping; physiologic management included delayed cord clamping. The Bristol trial evaluated 1695 women, with 849 randomly assigned to the active management group and 846 in the physiologic management group. The mean birth weight of the babies in the physiologic management group was $85 \mathrm{~g}$ higher than that of babies in the active group. ${ }^{36}$ The Hinchingbrooke trial involved 748 women in the active management of third stage group and 764 women in the physiologic management group. The infants in the physiologic management group had birth weights that averaged $67 \mathrm{~g}$ more than the infants in the active management of the third stage group. The authors attributed the difference to the additional blood that infants receive prior to cord clamping. ${ }^{37}$

\section{Infant Outcomes}

There are no studies comparing long-term outcomes of differences in cord clamping times. Suggested long-term impact on infants from premature ligation of the umbilical 

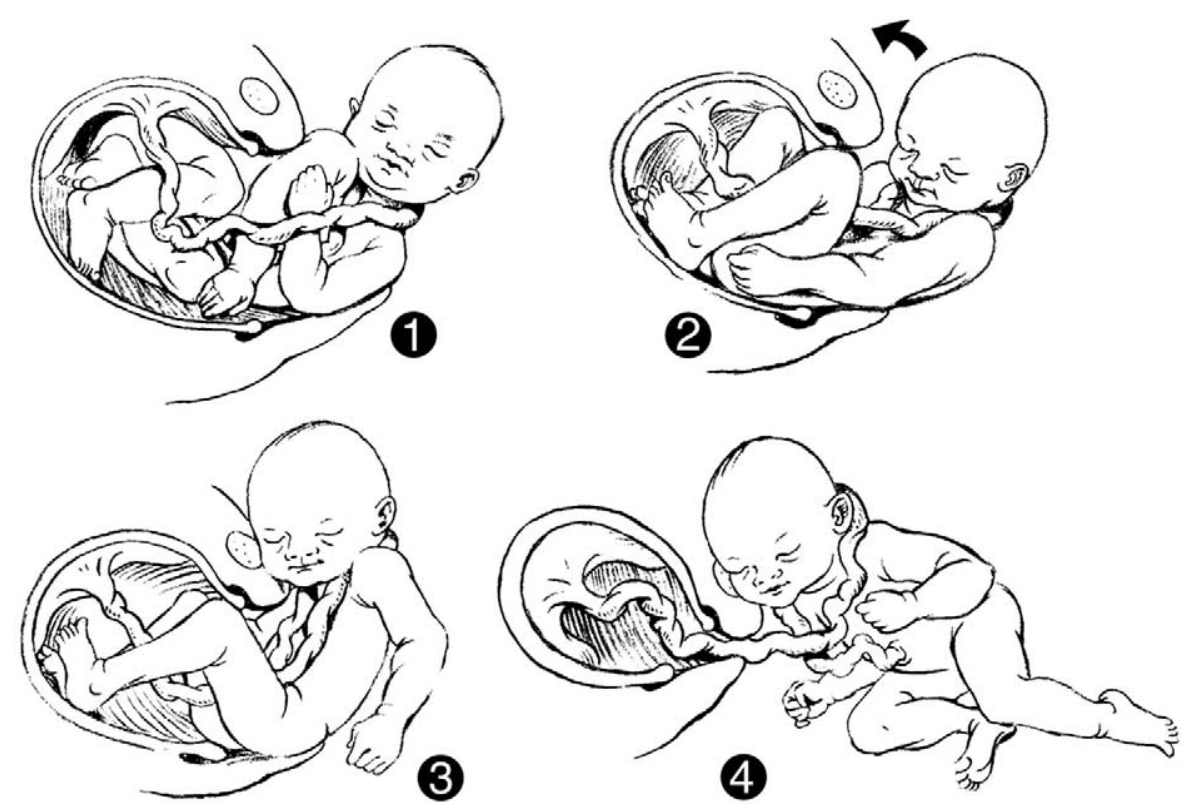

Figure 2. Somersault maneuver. The summersault maneuver involves holding the infant's head flexed and guiding it upward or sideways toward the pubic bone or thigh, so the baby does a "somersault," ending with the infant's feet toward the mother's knees and the head still at the perineum.

1. Once the nuchal cord is discovered, the anterior and posterior shoulders are slowly delivered under control without manipulating the cord.

2. As the shoulders are delivered, the head is flexed so that the face of the baby is pushed toward the maternal thigh

3. The baby's head is kept next to the perineum while the body is delivered and "somersaults" out.

4. The umbilical cord is then unwrapped, and the usual management ensues.

cord at birth or before birth includes anemia and neurodevelopmental delays. $^{32,38}$

A review of controlled trials of early versus late cord clamping at delivery evaluated the potential of delayed cord clamping to improve the iron status in infants at 2 to 3 months of age. ${ }^{32}$ The authors determined that late cord clamping at delivery significantly improved hemoglobin status in term infants at 2 to 3 months of age and did not increase adverse outcomes.

Clapp $^{38}$ conducted the only prospective study that followed infants who experienced a nuchal cord at birth until 1 year of age. He enrolled a group of 190 women who had normal pregnancies, attended the births, and completed developmental testing at 1 year of age in all of the infants. Sixty-six (35\%) of the women had infants with nuchal cords at birth. Twenty-one (11\%) had a nuchal cord at birth as an incidental finding with no signs during labor. However, 24\% were symptomatic for nuchal cord during labor, as evidenced by abnormal fetal heart rate patterns during labor or the finding of meconium-stained amniotic fluid at birth. At 1 year, scores on the Bayley Scales of Infant Development were significantly, albeit slightly, lower (116 versus 120 mental, and 101 versus 107 on psychomotor testing; $P<.01)$ in the infants who had experienced nuchal cords. This difference was more pronounced among the infants who had extreme tightness or multiple loops. No information on the management at birth was offered.

\section{LITERATURE ON MANAGEMENT OF NUCHAL CORD AT BIRTH}

No research was found on management of nuchal cords at birth, except for theoretical discussions, case reports, and a survey of midwifery practices. In 1991, Schorn and Blanco first described the somersault maneuver as a management option when a nuchal cord is present at a birth. ${ }^{10}$ It involves 1) slow delivery of the shoulders without manipulation of the cord, 2) flexing the neonate's head toward the mother's thigh as the shoulders are delivered, 3) keeping the infant's head close to the perineum and letting the body "somersault" out with feet pointing toward the mother's feet, and 4) unwrapping the cord and proceeding with normal management. The four maneuvers are described in Figure 2.

A survey of active members of the American College of Nurse-Midwives was conducted to inquire about cord clamping practices, beliefs, and nuchal cord management. ${ }^{39}$ Fifty-seven percent of the respondents chose the option "Clamp and cut the nuchal cord only when very tight." The somersault maneuver ${ }^{10}$ was selected by $40 \%$ of the participants as their best option for managing nuchal cord, and only $3.2 \%$ stated that they clamp and cut prior to birth of the shoulders in most cases of nuchal cord. Ninety-six percent of certified nurse-midwives surveyed avoid imme- 
diate clamping and cutting of the cord when confronted with a nuchal cord. ${ }^{39}$

\section{NUCHAL CORD MANAGEMENT: IMPLICATIONS FOR PRACTICE}

Maintaining the integrity of the nuchal cord as much as possible may decrease neonatal risks of hypovolemia, ${ }^{6}$ anemia, ${ }^{35}$ and hypoxic-ischemic encephalopathy, especially if birth is delayed by even a mild shoulder dystocia. ${ }^{1,8}$ Management of a presenting nuchal cord should be tailored to protect intact umbilical circulation. Techniques to preserve an intact nuchal cord depend on how tightly the cord is wrapped around the infant's neck. If the cord is loose, it can easily be slipped over the infant's head. The infant can be delivered normally and placed on maternal abdomen as desired. If the cord is too tight to go over the infant's head, the provider may be able to slip it over the infant's shoulders and deliver the body through the cord. The cord can then be unwrapped from around the baby after birth. Finally, if the cord is too tight to slip back over the shoulders, one may use the somersault maneuver, as described by Schorn and Blanco. ${ }^{10}$

\section{Resuscitation}

Resuscitation of the infant after reduction of a nuchal cord should take place between the mother's legs or on her abdomen, with the umbilical cord intact. Unwinding the cord allows the placental circulation to reperfuse the infant in the first minutes after birth so that the infant will not lose the essential blood volume necessary to oxygenate vital organs. ${ }^{27,28}$ Allowing time for this reperfusion while conducting resuscitation should improve the newborn's outcome.

Infants at the greatest risk of severe hypovolemia present with white, "drained" bodies (or mottled blue and white), no tone, no reflexes, and no respiratory efforts. However, they usually have heart rates above 100 . If the cord is left intact during resuscitation, they will reperfuse as the blood trapped in the placenta returns to the infant's body, correcting any acid-base imbalance that occurred in the process. Evidence for correction of the acid-base balance is that the tone returns about the same time that the baby begins breathing. ${ }^{11}$ If the heart rate is not above 100, drying (stimulation) and lowering the infant below the level of the perineum, ${ }^{40}$ or milking the cord $^{11}$ support resuscitative efforts. Bag and mask or even intubation can be done, as indicated, at the perineum (on a dry pad) without clamping the umbilical cord of the vulnerable infant. Once the infant has regained tone and color (reperfused) and breathing stabilizes, the infant can be put skin-to-skin on the mother's abdomen.

\section{SUMMARY AND CONCLUSION}

Nuchal cords occur in approximately $25 \%$ of births. Medical evidence indicates that clamping and cutting the cord prior to full delivery of the infant and/or immediately after birth increase the infant's risk of developing hypovolemia, anemia, hypovolemic shock, and rarely, cerebral palsy, if the birth is complicated by a shoulder dystocia after the cord has been cut. To provide a safe birth with minimal harmful intervention, obstetric providers need to fully understand how management impacts the normal physiologic processes of the newborn.

This literature review supports the use of the somersault maneuver with nuchal cord births. The maneuver, easily applied, is physiologically compatible with the normal fetus-to-infant transition processes and is based on a sound understanding of the form and function of the umbilical cord. The blood volume theory provides the theoretical rationale for the use of the somersault maneuver instead of clamping the cord before or immediately after birth.

A comprehensive inquiry into the normal physiologic fetus-to-newborn transitional process as well as the promotion of safe methods (i.e., somersault maneuver) for managing nuchal cords should be included in all midwifery and medical curricula. Educational and clinical preceptors can provide students with the opportunity to practice the somersault maneuver on dolls/models. Learning begins with awareness and exposure.

The current standard of practice is to immediately cut the cord to resuscitate the infant at the warmer. However, resuscitation at the perineum allows the infant to reperfuse and regain blood trapped in the placenta via postnatal placental circulation. In many institutions, responsibility for the mother-infant unit becomes divided at the time of birth. Obstetric and neonatal providers, with the best interest of the neonate at heart, can negotiate management to provide neonatal care that decreases, rather than increases, risk to the infant. Providers need to examine new views of neonatal transitional physiology in building rationale to avoid practices that have been shown to compromise the health of the infant.

This work was supported by the National Institutes of Health, National Institute for Nursing Research, Grant No. K 23 NR008027-01-02.

\section{REFERENCES}

1. Iffy L, Varadi V, Papp E. Untoward neonatal sequelae deriving from cutting of the umbilical cord before delivery. Med Law 2001; 20:627-34.

2. Dhar K, Ray S, Dhall G. Significance of nuchal cord. J Indian Med Assoc 1995;93:451-3.

3. Osak R, Webster K, Bocking A, Campbell M, Richardson B. Nuchal cord evident at birth impacts on fetal size relative to that of the placenta. Early Hum Dev 1997;49:193-202.

4. Larson J, Rayburn W, Harlan V. Nuchal cord entanglements and gestational age. Am J Perinatol 1997;14:555-7.

5. Cunningham F, McDonald $\mathrm{P}$, Gant $\mathrm{N}$, et al. Williams obstetrics. New York: McGraw-Hill, 2001:319-20. 
6. Cashore W, Usher R. Hypovolemia resulting from a tight nuchal cord at birth. Pediatr Res 1973;7:399.

7. Shepherd A, Richardson C, Brown J. Nuchal cord as a cause of neonatal anemia. Am J Dis Child 1985;139:71-3.

8. Iffy L, Varadi V. Cerebral palsy following cutting of the nuchal cord before delivery. Med Law 1994;13:323-30.

9. Nelson K, Grether J. Potentially asphyxiating conditions and spastic cerebral palsy in infants of normal birth weight. Am J Obstet Gynecol 1998;179:507-13.

10. Schorn M, Blanco J. Management of the nuchal cord. J Nurse Midwifery 1991;36:131-2.

11. Mercer J, Skovgaard R. Fetal to neonatal transition: First, do no harm. In Downe S, ed. Normal childbirth: Evidence and debate. Edinburgh: Elsevier, 2004:152.

12. Collins J. Tight nuchal cord morbidity and mortality. Am J Obstet Gynecol 1999;180:251.

13. Schaefer M, Laurichesse-Delmas H, Ville Y. The effect of nuchal cord on nuchal translucency measurement at 10-14 weeks. Ultrasound Obstet Gynecol 1998;11:271-3.

14. Vizza E, Correr S, Goranova V, et al. The collagen skeleton of the human umbilical cord at term. A scanning electron microscopy study after 2N-NaOH maceration. Reprod Fertil Dev 1996;8:885-94.

15. Reynolds S. Mechanism of placentofetal blood flow. Obstet Gynecol 1978;51:245-9.

16. Ball RH, Parer JT. The physiologic mechanisms of variable decelerations (discussion 1688-9). Am J Obstet Gynecol 1992;166: 1683-8.

17. Weiss E, Hitschold T, Berle P. Umbilical artery blood flow velocity waveforms during variable deceleration of the fetal heart rate. Am J Obstet Gynecol 1991;164:534-40.

18. Strong Jr, T Sarno A, Paul R. Significance of intrapartum amniotic fluid volume in the presence of nuchal cords. J Reprod Med 1992;37:718-20.

19. Mercer J, Skovgaard R. Neonatal transitional physiology: A new paradigm. J Perinat Neonatal Nurs 2002;15:56-75.

20. Blackburn S. Maternal, fetal, and neonatal physiology, 2nd ed. Philadelphia: WB Saunders, 2003:286-7.

21. Carlton D. Pediatrics and perinatology. In Gluckman P, Heyman M, eds. Pulmonary vasculature. London: Arnold, 1996:820.

22. Wardrop C, Holland B. The roles and vital importance of placental blood to the newborn infant. J Perinat Med 1995;23: $139-43$.

23. Yao A, Moinian M, Lind J. Distribution of blood between infant and placenta after birth. Lancet 1969;2:871-3.

24. Dunsmore S, Rannels D. Extracellular matrix biology in the lung. Am J Physiol 1996;270:L3-L27.
25. Jaykka S. Capillary erection and lung expansion; an experimental study of the effect of liquid pressure applied to the capillary network of excised fetal lungs. Acta Paediatr 1957;46:1-91.

26. Baier R, Hasan S, Cates D, Hooper D, Nowaczyk B, Rigatto H. Effects of various concentrations of $\mathrm{O}_{2}$ and umbilical cord occlusion on fetal breathing and behavior. J Appl Physiol 1990;68:1597-1604.

27. Stembera Z, Hodr J, Janda J. Umbilical blood flow in healthy newborn infants during the first minutes after birth. Am J Obstet Gynecol 1965;91:568-74.

28. Stembera Z, Hodr J, Janda J. Umbilical blood flow in newborn infants who suffered intrauterine hypoxia. Am J Obstet Gynecol 1968;101:546-53.

29. Saigal S, O’Neill A, Surainder Y, Chua LB, Usher R. Placental transfusion and hyperbilirubinemia in the premature. Pediatrics 1972; 49:406-19.

30. Saigal S, Usher R. Symptomatic neonatal plethora. Biol Neonate 1977;32:62-72.

31. Mercer J. Best evidence: A review of the literature on umbilical cord clamping. J Midwifery Womens Health 2001;46:402-14.

32. Rheenen P, Brabin B. Late umbilical cord-clamping as an intervention for reducing iron deficiency anaemia in term infants in developing and industrialised countries: A systematic review. Ann Trop Paediatr 2004;24:3-16.

33. Rabe H, Reynolds G, Diaz-Rossello J. Early versus delayed umbilical cord clamping in preterm infants. Cochrane Database Syst Rev 2004:CD003248.

34. Morley G. Cord closure: Can hasty clamping injure the newborn? Obg Management 1998:29-35.

35. Vanhaesebrouck P, Vanneste K, De Praeter C, Van Trappen Y, Thiery M. Tight nuchal cord and neonatal hypovolaemic shock. Arch Dis Child 1987;62:1276-7.

36. Prendiville W, Harding J, Elbourne D, Stirrat G. The Bristol third stage trial: Active versus physiological management of third stage of labour. BMJ 1988;297:1295-300.

37. Rogers J, Wood J, McCandlish R, Ayers S, Truesdale A, Elbourne D. Active versus expectant management of third stage of labour: The Hinchingbrooke randomised controlled trial. Lancet 1998;351:693-9.

38. Clapp III, J Lopez B, Simonean S. Nuchal cord and neurodevelopmental performance at 1 year. J Soc Gynecol Investig 1999;6: $268-72$.

39. Mercer J, Nelson C, Skovgaard R. Umbilical cord clamping: Beliefs and practices of American nurse-midwives. J Midwifery Womens Health 2000;45:58-66.

40. Yao A, Lind J. Effect of gravity on placental transfusion. Lancet 1969;2:505-8. 